\section{Photovoltaic Effects Exhibited in High-resistance Semi-conducting Films}

Microckystalime layers of lead sulphide have been obtained which, after suitable activation, exhibit photovoltaic effects of a new

type. photosensitive layer consists of a film about 1 micron in thickness deposited on glass between graphite electrodes a few millimetres apart. When illuminated by infra-red radiation of wave-length between approximately 1 and $3 \cdot 5$ microns, a photo E.M.F. is developed between the electrodes. It has been shown that this effect is independent of the contacts between the semi-conductor and the electrodes, and is due to the internal properties of the sensitive material. Cells with resistance of a few megohms and photo E.M.F. reaching 2 volts have been produced. These cells, as might be expected, show pronounced rectifying properties, the ratio of conductivities for opposite directions of current being about 100: 1 at room temperature. The photo current is always in the direction of high resistance.

The cells were produced as a result of information obtained during development work on the photoconductivity of activated thallium sulphide and lead sulphide layers. It is generally accepted that conductivity in semi-conductors is associated with the presence of impurity centres in the crystalline lattice. The impurity centres may be purity centres in the crystalline lattice. The impurity centres may be 'electronic' or 'bole' components of conductivity. Consideration of to 'electronic' or hole' components of conductivity. Consideration of ou restults obtained from sensitizing treatments in oxygen and in vacuo introduced, and further support is given to this by measurements of the Hall coefficient. High conductivity due to the presence of impurity of one sign alone is not associated with photosensitivity. The best photoconductivity in a given layer appears when maximum resistance is obtained, which may be explained by the existence of some kind of equilibrium between impurity centres of both signs which are simultaneously present. Also the experimental results are best explained by the assumption that the positive and negative centres are not uniformly distributed and that variations in relative concentration are closely associated with the microcrystalline structure of the layer, some of the crystals having a concentration of centres of a given sign different from their neighbours.

A semi-quantitative model was developed which postulated the existence of barriers at the boundaries of the crystals, with unequal concentration of impurity centres on opposite sides. The properties of normal photoconducting layers could be explained by random orientation and distribution of these barriers, but the possibility also arose of orientating them to produce a resultant photovoltaic effect from the cell. Such a cell, because of the superposition of the effects of many individual elements in series, should be strongly rectifying and, unlike the usual photovoltaic cells, should have a high resistance and high voltage sensitivity.

The treatment which was successful in producing photovoltaic cells of this kind consisted in passing a current through the photoconducting film at a temperature sufficiently high to ensure mobility of oxygen ions (about $250^{\circ}$ C.). The direction of the resultant photo E.M.F. is in the opposite sense to the activating current. Layers treated in this way also show a marked directional photoconductivity; the conductivify in the high-resistance direction is strongly increased by illumination, and in the low-resistance direction the increase is less pronounced.

Under certain conditions, non-uniform activation across the cell may be obtained; and it is found that the photovoltaic and photoconductive sensitivity are always associated with the same part of the layer, as would be expected from the above considerations. Also towards the positive electrode, indicating the mobility of negative centres and a fairly uniform distribution of positive centres.

A full description of the experimental work and a more detailed development of the theoretical considerations will be given elsewhere.

$\begin{array}{ll} & \text { J. STARKIEWIC/ } \\ \text { L. SOSNOWSK1 } & \text { O. SIMPSON }\end{array}$

Admiralty Research Laboratory,

o. SIMPSON

Teddington, Middlesex.

May 25.

\section{Surface Area Determination}

In the determination of surface area using nitrogen at the temperature of liquid nitrogen by the method of Brunauer, Emmett and ture of liquid nitrogen by the method of Brunauer, Emmett and
Teller $^{1}$, it is usual to determine the dead-space on each sample, using Teller ${ }^{1}$, it is usual to determine the dead-space on each sample, using
helium. For routine determinations on a substance known to give $S$-shaped isotherms, that is, where the equation of Brunauer, Emmett $S$-shaped isotherms, that is, where the equation of Brunauer, Emmett
and Teller is applicable, it is possible to avoid this dead-space determand Teller is applicable, it is possible to avoid this dead-space determ-
ination. If $V_{1}$ c.c. nitrogen are adsorbed at a pressure $p_{1}$, then the ination. If $V_{1}$ e.c. nit
dead-space is given by

$$
p_{1}(1+\alpha) D \bumpeq p_{1} D
$$

where $D$ is dead-space at unit pressure and $\alpha$ is a correction factor equal to $6.58 \times 10^{-5}$ at unit pressure. Hence, if $V_{c_{1}}$ is volume of nitrogen in the sample bulb, then

$V_{1}=V_{c_{1}}-p_{1} D ; \quad V_{2}=V_{c_{2}}-p_{2} D ; \quad V_{3}=V_{c_{3}}-p_{3} D$. It follows that

$$
V_{1}=V_{c_{1}}-p_{1}\left(\frac{V_{c_{3}}-V_{3}}{p_{3}}\right)
$$

Emmett ${ }^{2}$ points out that a line drawn through the origin and one adsorption point at a relative pressure of $c \cdot 0 \cdot 3$ usually differs in slope by less than 5 per cent from that drawn using several adsorption points.
If this is assumed, then

$$
V_{1}\left(p_{0}-p_{1}\right)=V_{2}\left(p_{3}-p_{2}\right)=V_{3}\left(p_{0}-p_{3}\right) \text {. }
$$

Therefore

$$
V_{1}=V_{c_{1}}-\frac{p_{1}}{p_{3}}\left(V_{c_{3}}-V_{1} \cdot \frac{p_{0}-p_{1}}{p_{0}-p_{3}}\right) .
$$

Rearranging,

$$
V_{1}=\frac{V_{c_{1}}-V_{c_{3}} \frac{p_{2}}{p_{3}}}{1-\frac{p_{1}}{p_{3}}\left(\frac{p_{0}-p_{1}}{p_{0}-p_{3}}\right)}
$$

and similarly for $V_{2}$.

In the plot of $p / p_{0}$ against $\frac{p}{V\left(p_{0}-\bar{p}\right)}$, the monolayer volume $V_{m}$ is the reciprocal of the slope, that is,

$$
V_{m}=\frac{\left(p_{2}-p_{1}\right) / p_{0}}{p_{2} /\left\{V_{2}\left(p_{0}-p_{2}\right)\right\}-p_{1} /\left\{V_{1}\left(p_{0}-p_{1}\right)\right\}}
$$

This involves determining three adsorption points, but no dead-space : a slightly less accurate value can be found using only two points, that is,

$$
V_{m}=\frac{p_{1} / p_{0}}{p_{1} /\left\{V_{1}\left(p_{0}-p_{1}\right)\right\}}=\frac{V_{1}}{p_{0}}\left(p_{0}-p_{1}\right) .
$$

In neither case is it necessary actually to plot the points. Areas determined on various substances by the usual technique, that is, involving a dead-space determination, are compared with those obtained by recalculation using equation (1) above in the following table:

\begin{tabular}{|l|c|c|}
\hline \multirow{2}{*}{ Substance } & \multicolumn{2}{|c|}{ Area in sq. m./gm. } \\
\cline { 2 - 3 } & $\begin{array}{c}\text { By dead-space } \\
\text { determination }\end{array}$ & By recalculation \\
\hline Carbon $A$ & 790 & 753 \\
Carbon $B$ & 847 & 840 \\
Carbon $C$ & 814 & 800 \\
Carbon $D$ & 460 & 460 \\
Carbon $E$ & 280 & 282 \\
Chalk $A$ & 30 & 29 \\
Chalk $B$ & 19 & 19 \\
Paris White & 2 & 2 \\
Clay & 10 & 10 \\
Asbestos & 18 & 14 \\
\hline
\end{tabular}

It will be noted that the calculated areas are generally lower than the dead-space areas, that is, the slopes are too great: this is in accordance with the assumption made above.

Our thanks are due to the directors of the Washington Chemical Company, Ltd., for permission to publish this note.

$$
\begin{aligned}
& \text { P. E. BUGGE } \\
& \text { R. H. KERLOGUE } \\
& \text { F. WESTWICK }
\end{aligned}
$$

Research Laboratories,

The Washington Chemical Co., Ltd.,

Washington, Co. Durham.

${ }^{1}$ Brunauer, Emmett and Teller, J. Amer. Chem. Soc., 60, 309 (1938). Emmett, P. H., "Symposium on New Methods of Determining
Particle Size in Subsieve Range", Amer. Soc. Test. Mat., p. 95 Particle Size
(March 1941).

\section{An Optical-Acoustic Method of Gas Analysis}

Bell, Tyndall and Röntgen all knew that gases and vapours absorb infra-red rays and that they would produce sounds if a stream of rays was interrupted at sonic frequencies. The mechanism of this phenomenon is as follows. When a gas absorbs infra-red rays, it is phenomenon is as follows. When a gas absorbs infra-red rays, it is rupted at sonic frequency, there are rapid changes of pressure in the gas. Pulsation takes place, which is nothing more nor less than sound. The pitch of the sound depends on the frequency at which the rays are interrupted, and its strength on the ability of the gas to absorb infra. interrupted, and its strength on the ability of the gas to absorb infrared rays. If pure air, in which there are no extraneous gases or vapours, is submitted to interrupted irradiation with infra-red rays, there will be no sound, since oxygen and nitrogen, of which the air is composed, do not absorb infra-red rays. If the rays are passed through a mixture of air and a gas or vapour, the sound produced will be loude greater the quantity of extraneous matter present in the air.
Tyndall first spoke of using this phenomenon to detect gases in Tyndall first spoke of using this phenomenon to detect gases in
mines so early as 1888 . He had at his disposal no instrument with which to measure the strength of sounds, and could not therefore put his idea into practice. The idea of using interrupted infra-red rays for this purpose was forgotten until 1937, when I developed my optical-acoustic gas analyser, involving the use of modern reception and measuring instruments. This analyser will determine the quantitative composition of a gas mixture the qualitative content of which is already known. The method is almost universal and will detect all gases except oxygen, hydrogen and nitrogen, which do not absorb infra-red rays.

The principle of the analyser is, briefly, as follows: Infra-red rays box with a window through which rays pass. They are inter- 